University of Nebraska - Lincoln

DigitalCommons@University of Nebraska - Lincoln

Faculty Publications from the Department of Engineering Mechanics

$10-15-2008$

\title{
Contact-resonance atomic force microscopy for viscoelasticity
}

\author{
P. A. Yuya \\ University of Nebraska - Lincoln \\ D. C. Hurley \\ Materials Reliability Division, National Institute of Standards and Technology, Boulder, Colorado \\ Joseph A. Turner \\ University of Nebraska - Lincoln, jaturner@unl.edu
}

Follow this and additional works at: https://digitalcommons.unl.edu/engineeringmechanicsfacpub

Part of the Mechanical Engineering Commons

Yuya, P. A.; Hurley, D. C.; and Turner, Joseph A., "Contact-resonance atomic force microscopy for viscoelasticity" (2008). Faculty Publications from the Department of Engineering Mechanics. 12. https://digitalcommons.unl.edu/engineeringmechanicsfacpub/12

This Article is brought to you for free and open access by the Mechanical \& Materials Engineering, Department of at DigitalCommons@University of Nebraska - Lincoln. It has been accepted for inclusion in Faculty Publications from the Department of Engineering Mechanics by an authorized administrator of DigitalCommons@University of Nebraska - Lincoln. 


\title{
Contact-resonance atomic force microscopy for viscoelasticity
}

\author{
P. A. Yuya, ${ }^{1}$ D. C. Hurley, ${ }^{2}$ and J. A. Turner ${ }^{1, a)}$ \\ ${ }^{1}$ Department of Engineering Mechanics, University of Nebraska-Lincoln, W317.4 Nebraska Hall, \\ Lincoln, Nebraska 68588-0526, USA \\ ${ }^{2}$ Materials Reliability Division, National Institute of Standards and Technology, \\ Boulder, Colorado 80305, USA
}

(Received 3 June 2008; accepted 20 August 2008; published online 15 October 2008)

\begin{abstract}
We present a quantitative method for determining the viscoelastic properties of materials with nanometer spatial resolution. The approach is based on the atomic force acoustic microscopy technique that involves the resonant frequencies of the atomic force microscopy cantilever when its tip is in contact with a sample surface. We derive expressions for the viscoelastic properties of the sample in terms of the cantilever frequency response and damping loss. We demonstrate the approach by obtaining experimental values for the storage and loss moduli of a poly(methyl methacrylate) film using a polystyrene sample as a reference material. Experimental techniques and system calibration methods to perform material property measurements are also presented. (C) 2008 American Institute of Physics. [DOI: 10.1063/1.2996259]
\end{abstract}

\section{INTRODUCTION}

Knowledge of viscoelastic properties at the nanoscale is essential for the development of advanced materials and devices with applications in various areas of nanotechnology. However, properties measured from bulk specimens may not accurately represent those of the nanomaterial. Because the small length scales prevent the use of many well-established measurement techniques, other characterization approaches must be developed. Atomic force microscopy ${ }^{1}$ (AFM) has become a valuable tool for studying the mechanical response of materials at the nanoscale. AFM approaches can be used, for example, for topography and stiffness imaging ${ }^{2,3}$ to evaluate tribological properties of thin films, ${ }^{4}$ force measurements, ${ }^{5}$ and adhesion energy. ${ }^{6}$ By modeling the tipsample interaction as two springs in series, the mechanical response of the sample can be determined from the contact portion of the resulting force curve. ${ }^{7}$ Rosa-Zeiser et al. ${ }^{8}$ used the pulsed-force mode of the scanning force microscope to image elastic, electrostatic, and adhesive properties simultaneously with topography. By modeling the tip-sample interaction using a spring-dashpot system, Burnham et al. ${ }^{9}$ were able to image local energy dissipation through impacts using a scanning probe microscopy method. Viscoelastic relaxation can be measured with AFM by determining the dissipation energy from the hysteresis between the loading and unloading segments of the force curve. ${ }^{10}$

The work of Attard ${ }^{10}$ provided an excellent overview of the extensive research of late regarding viscoelasticity at nanometer length scales. In spite of the variety of AFM imaging methods developed, none has been used to determine viscoelastic properties quantitatively. Here, we show how nanoscale viscoelastic properties of materials, namely, the storage and loss moduli, can be determined using contact-

\footnotetext{
${ }^{a)}$ Author to whom correspondence should be addressed. Tel.: (402) 4728856. Electronic mail: jaturner@unl.edu.
}

resonance AFM for viscoelasticity (CRAVE). The CRAVE technique is based on the atomic force acoustic microscopy (AFAM) method of contact-resonance AFM and has similarities with the nanoDMA ${ }^{\circledR}$ approach (Hysitron, Minneapolis, $\mathrm{MN}) .{ }^{11}$ In both techniques, the displacement amplitude and phase shift between the excitation force and displacement are measured using a lock-in amplifier. The contact stiffness is calculated from the amplitude and phase shift by making use of an appropriate contact model. In quantitative AFAM, ${ }^{2,12}$ a transducer below the sample generates out-of-plane vibrations that excite the resonant modes of the AFM cantilever when the tip is in contact. Measurements of the resonant frequencies are analyzed with analytical models in order to obtain the elastic properties of the sample. ${ }^{13}$ With few exceptions, ${ }^{14,15}$ AFAM studies have been limited to modeling the tip-sample interaction as a linear spring. Dissipation due to damping and adhesion ${ }^{16}$ is normally ignored.

Including a dashpot in the contact-resonance AFM model of the tip-sample interaction allows the viscoelastic properties of the sample to be determined. Here, we show how the complex wavenumbers of the resonant system are defined and separated in order to determine the contributions of the elastic components and the dissipation due to damping. Expressions are derived for the amplitude and phase angle of the frequency response of a cantilever beam excited by a harmonic force. Experimental contact-resonance spectra are then fit to the predicted response in order to determine values for the loss and storage moduli. The CRAVE technique demonstrates how contact-resonance AFM methods can provide information about viscoelastic properties in cases where indenter-tip size limitations prevent the use of nanoDMA techniques. Furthermore, the contact loads applied with CRAVE can be limited to nanonewtons, thus reducing or eliminating sample damage and minimizing potential effects such as enhanced stiffness due to confinement (an effect still under investigation). ${ }^{17}$ 


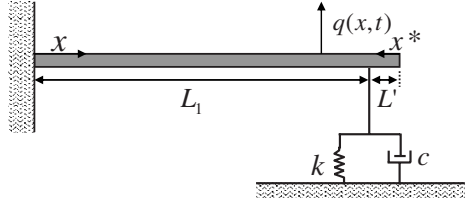

FIG. 1. An AFM tip in contact with a viscoelastic surface. The tip-sample forces are approximated by a linear spring-dashpot system.

\section{THEORETICAL MODEL}

Figure 1 depicts an AFM cantilever in contact with a viscoelastic surface. Typically, an AFM cantilever is tilted at an angle $\left(\theta_{0} \approx 15^{\circ}\right)$ relative to the sample surface. ${ }^{18}$ As a result, cantilever forces applied in the vertical direction therefore have a small component parallel to the sample surface lowering the contact frequencies slightly. For simplicity, here we assume the cantilever to be aligned parallel to the sample surface $\left(\theta_{0}=0\right)$. The cantilever is assumed to be a rectangular beam with width $w$, thickness $b$, and length $L$. The tip-sample interaction is represented by a spring of stiffness $k$ and a dashpot with characteristic damping $c$ that depends on the frequency. The position of the AFM tip from the clamped end is represented by $L_{1}$, while the length of the beam from the tip to the unclamped end is $L^{\prime}$. The forces between the tip and the sample surface are modeled using a Kelvin-Voigt mechanical equivalent. The governing equation for flexural vibrations for this system is expressed as ${ }^{19}$

$$
E I \frac{\partial^{4} q(x, t)}{\partial x^{4}}+\chi \frac{\partial q(x, t)}{\partial t}+A \rho \frac{\partial^{2} q(x, t)}{\partial t^{2}}=0
$$

where $q(x, t)$ is the cantilever displacement, $E$ is the Young's modulus, $I$ is the bending moment of inertia, $\rho$ is the density of the cantilever material, $A$ is the cross-sectional area, and $\chi$ represents damping in the beam. The cantilever is divided into two parts with lengths $L_{1}$ and $L^{\prime}$ with separate harmonic solutions $q(x, t)=\Omega(x) e^{i \omega t}$ and $q^{*}\left(x^{*}, t\right)=\Omega^{*}\left(x^{*}\right) e^{i \omega t}$, respectively. Here $q^{*}\left(x^{*}, t\right)$ is the displacement from the free end of the cantilever, the $x^{*}$-axis is defined in the negative $x$-axis direction, and $\omega$ is the angular frequency. The geometric and natural boundary conditions for the problem dictate zero slope and displacement at the fixed end, and zero bending moment and shear force at the free end. The continuity conditions at the coupling position $\left(x=L_{1}\right)$ ensure that the displacement, slope, and bending moment are equal, while the shear force in the beam is balanced by the forces due to contact stiffness and contact damping. The characteristic equation obtained by satisfying the boundary and continuity conditions can be expressed as ${ }^{14}$

$$
\begin{aligned}
\frac{2}{3}\left(\lambda_{n} L_{1}\right)^{3}\left[1+\cos \lambda_{n} L_{1} \cosh \lambda_{n} L_{1}\right] \\
=\left[\alpha+i \beta\left(\lambda_{n} L_{1}\right)^{2}\right]\left[\left(1+\cos \lambda_{n} L^{\prime} \cosh \lambda_{n} L^{\prime}\right)\right. \\
\quad \times\left(\sinh \lambda_{n} L_{1} \cos \lambda_{n} L_{1}-\sin \lambda_{n} L_{1} \cosh \lambda_{n} L_{1}\right) \\
\quad+\left(1-\cos \lambda_{n} L_{1} \cosh \lambda_{n} L_{1}\right)\left(\sin \lambda_{n} L^{\prime} \cosh \lambda_{n} L^{\prime}\right. \\
\left.\left.\quad-\cos \lambda_{n} L^{\prime} \sinh \lambda_{n} L^{\prime}\right)\right],
\end{aligned}
$$

where $\alpha=k L^{3} / 3 E I=k / k_{c}, k_{c}$ is the cantilever stiffness, and $\beta=c \sqrt{L^{2} /(9 E I \rho A)}$. In our analysis, we quantify the viscoelastic properties of the sample by the complex wavenumbers $\lambda_{n} L_{1}=\left(a_{n}+i b_{n}\right)$, where $a$ represents the stiffness of the tip-sample configuration, $b$ represents the viscous damping behavior of the sample, and $n=1,2 . . \infty$ are the mode numbers. The values of $a$ and $b$ are determined by fitting the experimental frequency response. The complex characteristic Eq. (2) is then solved numerically for $\alpha$ and $\beta$ once the values of $a$ and $b$ are obtained.

\section{RESPONSE TO HARMONIC EXCITATION}

In this section, we look at the response of an AFM cantilever beam to harmonic excitation to determine expressions for displacement amplitude and phase that are used to fit to experimental data to determine the parameters $a$ and $b$. If a harmonic force $F e^{i \omega t}$ is applied to the beam at $x=x_{0}$, Eq. (1) becomes

$$
E I \frac{\partial^{4} q(x, t)}{\partial x^{4}}+\chi \frac{\partial q(x, t)}{\partial t}+A \rho \frac{\partial^{2} q(x, t)}{\partial t^{2}}=F \delta\left(x-x_{0}\right) e^{i \omega t},
$$

where $\delta(z)$ is Dirac's delta function. Equation (3) is solved by superposition of the natural modes of the unforced system. The displacement can then be expressed as

$$
q(x, t)=e^{i \omega t} \sum_{n=1}^{\infty} P_{n} Y_{n}(x),
$$

where the $P_{n}$ coefficients give the mode weighting, and $Y_{n}(x)$ are the spatial eigenfunctions determined from the analysis of a clamped beam in free vibration as ${ }^{20}$

$$
\begin{aligned}
Y_{n}(x)= & \left(\frac{\sin \left(\lambda_{n} L\right)-\sinh \left(\lambda_{n} L\right)}{\cos \left(\lambda_{n} L\right)+\cosh \left(\lambda_{n} L\right)}\right)\left[\sin \left(\lambda_{n} x\right)-\sinh \left(\lambda_{n} x\right)\right] \\
& +\left[\cos \left(\lambda_{n} x\right)-\cosh \left(\lambda_{n} x\right)\right] .
\end{aligned}
$$

The wavenumbers $\lambda_{n}$ depend on the resonant frequency. Substituting Eq. (4) into Eq. (3) gives

$$
\left[E I \lambda_{n}^{4}-\rho A \omega^{2}+i \omega \chi\right] P_{n} Y_{n}(x)=F \delta\left(x-x_{0}\right) .
$$

Because the modes are orthogonal, Eq. (6) can be multiplied by $Y_{m}(x)$ and integrated from 0 to $L$ to determine the constants $P_{n}$; thus

$$
\begin{aligned}
\sum_{n=1}^{\infty} & {\left[E I \lambda_{n}^{4}-\rho A \omega^{2}+i \omega \chi\right] \int_{0}^{L} P_{n} Y_{n}(x) Y_{m}(x) d x } \\
& =\int_{0}^{L} F Y_{m}(x) \delta\left(x-x_{0}\right) d x .
\end{aligned}
$$

For an orthonormal set of functions we have ${ }^{21}$ $\int_{0}^{L} Y_{n}(x) Y_{m}(x) d x=L \delta_{m n}$, which allows Eq. (7) to be integrated, resulting in $P_{n}=F Y_{n}\left(x_{0}\right) / L\left[E I \lambda_{n}^{4}-\rho A \omega^{2}+i \omega \chi\right]$. The response of the beam to harmonic excitations at the end of the beam can then be expressed as

$$
q(x, t)=\sum_{n=1}^{\infty} \frac{F Y_{n}(L) e^{i \omega t}}{m_{b}\left[N \gamma_{n}^{4}-\omega^{2}+i \omega \bar{\chi}\right]} Y_{n}(x),
$$

where $m_{b}=\rho A L$ is mass of the beam, $N=E I / m_{b} L^{3}$ and $\bar{\chi}$ $=\chi / \rho A$ are beam constants, while $\gamma_{n}=\lambda_{n} L$ are the normal- 
ized wavenumbers. If we take the tip to be at the very end of the cantilever $\left(L_{1}=L\right)$, Eq. (8) becomes

$$
q(L, t)=\sum_{n=1}^{\infty} \frac{F Y_{n}^{2}(L) e^{i \omega t}}{m_{b}\left[N \gamma_{n}^{4}-\omega^{2}+i \omega \bar{\chi}\right]} .
$$

From Eq. (5), $Y_{n}(L)$ can be expressed as $Y_{n}(L)=2(1$ $\left.-\sin \gamma_{n} \sinh \gamma_{n}\right) /\left(\cos \gamma_{n}+\cosh \gamma_{n}\right)$. Therefore

$$
q(L, t)=\sum_{n=1}^{\infty} \frac{F e^{i \omega t}}{m_{b}\left[N \gamma_{n}^{4}-\omega^{2}+i \omega \bar{\chi}\right]}\left(\frac{2\left(1-\sin \gamma_{n} \sinh \gamma_{n}\right)}{\cos \gamma_{n}+\cosh \gamma_{n}}\right)^{2} .
$$

For the first mode $(n=1)$, the frequency response of the system is expressed as

$$
\begin{aligned}
G(i \omega)= & \frac{q(L, t) m_{b}}{F} \\
= & \frac{1}{\left[N(a+i b)^{4}-\omega^{2}+i \omega \bar{\chi}\right]} \\
& \quad \times\left\{\frac{2[1-\sin (a+i b) \sinh (a+i b)]}{\cos (a+i b)+\cosh (a+i b)}\right\}^{2},
\end{aligned}
$$

such that $|G(i \omega)|=\sqrt{\{\operatorname{Re}[G(i \omega)]\}^{2}+\{\operatorname{Im}[G(i \omega)]\}^{2}}$. The solution for the displacement at the end of the beam can be expressed as $q(L, t)=D \cos (\omega t-\phi)$, where $D=F / m_{b}|G(i \omega)|$ is the displacement amplitude. The phase shift between the applied force and measured displacement is

$$
\phi=\tan ^{-1}[-\operatorname{Im} G(i \omega) / \operatorname{Re} G(i \omega)] .
$$

When the tip is not in contact with the specimen surface, there is no moment or shear force supported at the free end $(x=L)$. The wavenumbers for the free vibration of the beam are obtained by solving the characteristic equation resulting from these boundary conditions: $:^{22} \cos \gamma \cosh \gamma+1=0$. For the free case, we have $b=0$ such that $\gamma=a$. Therefore Eq. (11) reduces to

$$
G_{\text {free }}(i \omega)=\frac{1}{\left[N a^{4}-\omega^{2}+i \omega \bar{\chi}\right]}\left(\frac{2(1-\sin a \sinh a)}{\cos a+\cosh a}\right)^{2} .
$$

The values of $N$ and $\bar{\chi}$ in Eq. (13) are obtained from the free frequency spectrum by making use of the known values for $a$ (1.8751, 4.6941, 7.8547, etc.).

\section{AFM TIP-SAMPLE CONTACT MECHANICS}

The mechanics of the AFM tip-sample contact gives the connection between the experimentally measured values and the sample mechanical properties such as modulus. The contact mechanics for AFM tips is very difficult to model because the exact shape of the tip in contact with the sample is usually unknown. The most commonly used is the Hertzian contact model. ${ }^{23}$ For a Hertzian model, a spherical indenter with radius $R$ contacting a flat surface with a normal force $F_{N}$ forms a contact radius given by ${ }^{23} a_{c}=\sqrt[3]{3 F_{N} R / 4 E^{*}}$, where $E^{*}$ is the reduced Young's modulus given by $1 / E^{*}=1 / M_{t}$ $+1 / M_{s}$. Here $M$ is the indentation modulus given by $M$ $=E /\left(1-\nu^{2}\right)$, where $E$ is the Young's modulus and $\nu$ is Pois-

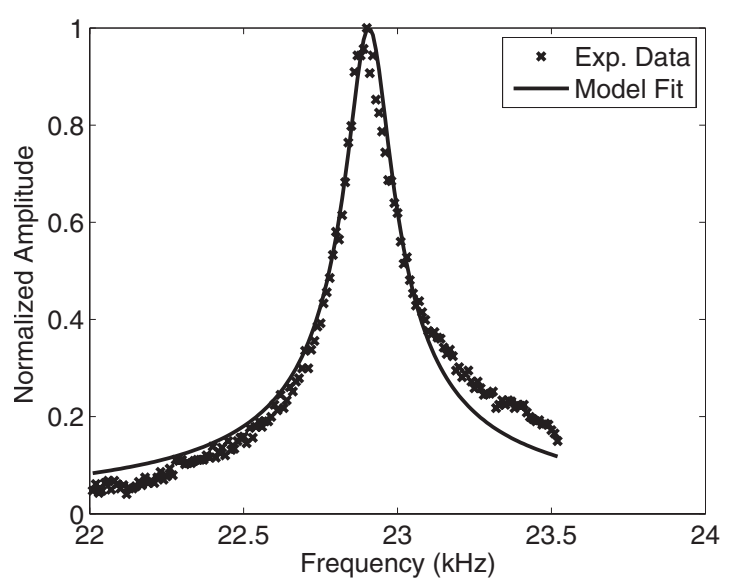

FIG. 2. Frequency response of the AFM cantilever vibrating out of contact with the sample.

son's ratio. The subscripts $t$ and $s$ refer to the AFM tip and the sample, respectively. The reduced complex modulus of a linear viscoelastic material is given by $E^{*}(\omega)=E^{\prime *}+i E^{\prime \prime *}$. Here, $E^{\prime *}$ is the reduced storage modulus, which is in phase with the strain and represents the elastic behavior, while $E^{\prime \prime *}$ is the reduced loss modulus and represents the internal damping. Using the elastic solution for the Young's modulus from nanoindentation and the elastic-viscoelastic correspondence principle, ${ }^{24}$ the reduced storage modulus of a viscoelastic material determined by nanoindentation is expressed as $E^{\prime *}=1 / 2 k \sqrt{\pi / A}$, where $A$ is the contact area obtained from the tip area function. Similarly, the loss modulus is expressed as $E^{\prime \prime *}=1 / 2 \omega c \sqrt{\pi / A}$. Because accurate determination of the AFM contact area is extremely difficult, AFAM methods use an alternative approach involving a reference material with known properties. ${ }^{2,13}$ The reduced storage and loss moduli of the unknown sample are then expressed in terms of the reference sample as $E_{s}^{\prime *}$ $=E_{\mathrm{ref}}^{\prime *}\left(k_{s} / k_{\mathrm{ref}}\right)^{m}$ and $E_{s}^{\prime \prime *}=E_{\mathrm{ref}}^{\prime \prime *}\left(\omega_{s} c_{s} / \omega_{\mathrm{ref}} c_{\mathrm{ref}}\right)^{m}$ where the subscript "ref" refers to a reference sample, $\omega$ is the frequency, and $m$ is a constant that depends on the tip-sample geometry. For Hertzian contact, $m=3 / 2$, while for a flat tip, $m=1$.

\section{EXPERIMENTAL METHODS AND DATA ANALYSIS}

In the CRAVE technique (as with other contactresonance approaches), an AFM cantilever beam is set into flexural oscillations by out-of-plane movement of the sample caused by ultrasonic waves. The sample to be investigated is mounted on a transducer, which is excited with a continuous sine wave voltage from a function generator. The amplitude of the cantilever oscillations is detected by the standard optical beam-deflection scheme of an AFM. A lock-in amplifier is used to detect the amplitude and phase of the AFM photodiode signal at the transducer frequency. A spectrum of the cantilever response versus frequency is obtained by sweeping the transducer excitation frequency and recording the lock-in output signal. The resonant frequency and the shape of the frequency spectra depend on the complex wavenumbers corresponding to the various vibrational modes of the cantilever beam. 

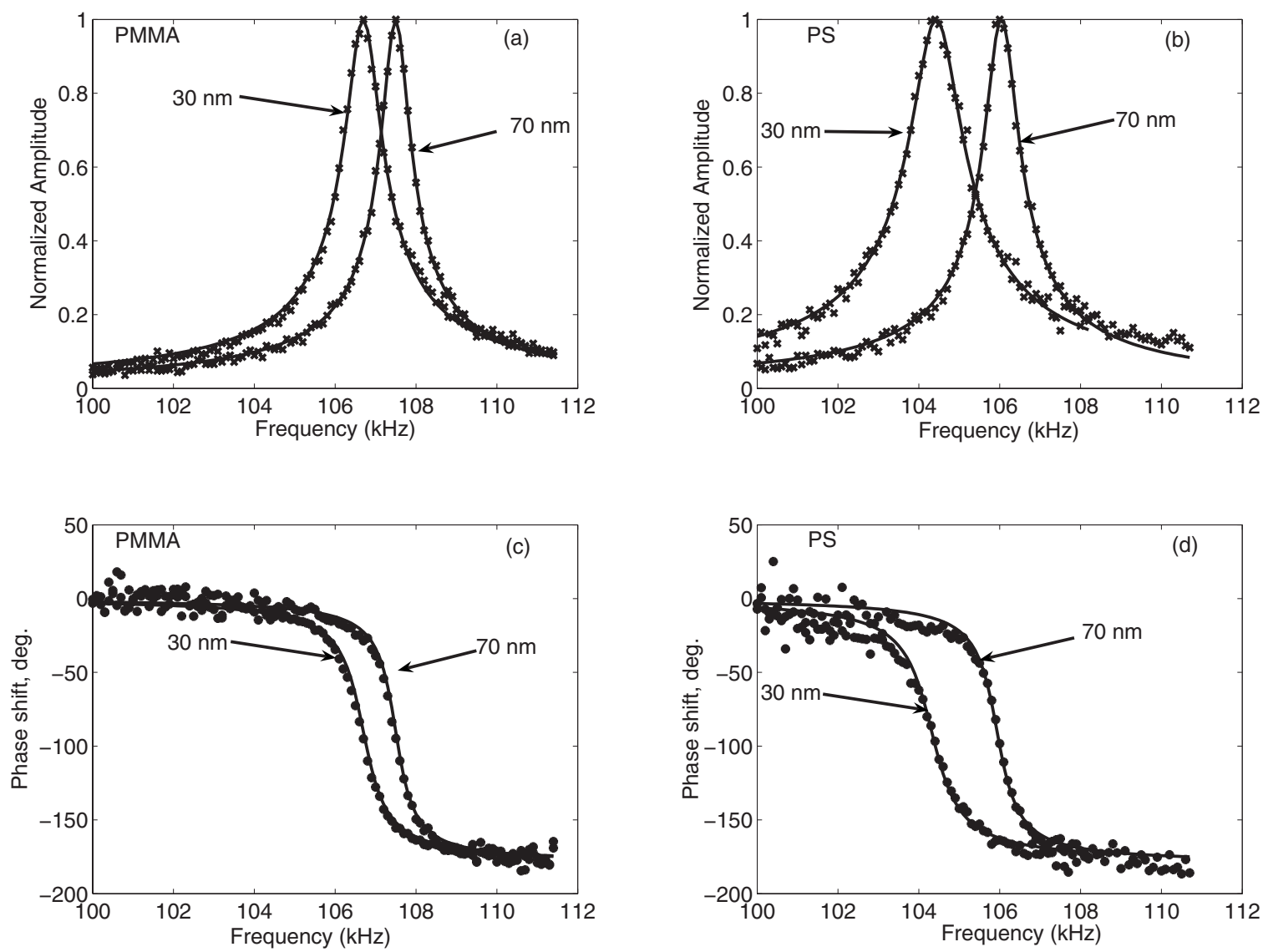

FIG. 3. Example of first mode response of the cantilever for the tip in contact with the PMMA film and the reference sample (PS). (a) and (b) show the amplitude spectra for PMMA and PS, respectively. Respective phase spectra are shown in (c) and (d). The solid line shows the fit to the model given by Eqs. (11) and (12).

To study the damping behavior of the sample, it is important first to evaluate the damping of the beam when vibrating with the tip out of contact. This is mainly because the damping measured when in contact is a combined response of the beam and the sample. Using Eq. (13), a nonlinear least-squares fit is performed on the measured free-space amplitude and phase response to obtain values for $N$ and $\bar{\chi}$. Next, contact-resonance frequency measurements are made on both the test sample with unknown properties and the calibration sample whose properties have been determined independently. Using Eq. (11), a nonlinear least-squares fit is performed on the amplitude spectra to determine the values of $a$ and $b$ of the complex wavenumber. These values of $a$ and $b$ are then used to determine numerically the values of $\alpha$ and $\beta$ from the characteristic equation [Eq. (2)]. Finally, from the values of $\alpha$ and $\beta$ and independent knowledge of the storage and loss moduli of the reference sample, the storage modulus $E^{\prime}$ and the loss modulus $E^{\prime \prime}$ of the test sample can be obtained. This is accomplished using modified versions of the equations relating the storage and loss moduli of the unknown sample to those of the reference sample as $E_{s}^{\prime *}=E_{\text {ref }}^{\prime *}\left(\alpha_{s} / \alpha_{\text {ref }}\right)^{m}$ and $E_{s}^{\prime \prime *}=E_{\text {ref }}^{\prime \prime *}\left(\omega_{s} \beta_{s} / \omega_{\text {ref }} \beta_{\text {ref }}\right)^{m}$.

In our experiments, we used a rectangular, single-crystal silicon cantilever. The nominal properties of the cantilever were length $L=450 \mu \mathrm{m}$, width $w=50 \mu \mathrm{m}$, thickness $b$ $=4 \mu \mathrm{m}$, and stiffness $k_{c}=1 \mathrm{~N} / \mathrm{m}$. Figure 2 shows the amplitude response for the cantilever in free space. From the fit, the free resonance of the cantilever is $f=22.90 \mathrm{kHz}$, the beam damping $\bar{\chi}=\chi / \rho A$ is $(1.46 \pm 0.05) \times 10^{2} \mathrm{~s}^{-1}$, and the constant $N$ that depends on the beam properties is $(4.2249 \pm 0.0161) \times 10^{7} \mathrm{~s}^{-2}$. These values represent the average and standard deviation of six curve fits, corresponding to two different frequency spectra for each of the first three free flexural modes.

The test sample for the CRAVE experiments was a film of poly(methyl methacrylate) (PMMA) approximately 890 $\mathrm{nm}$ thick on a (001) silicon substrate. The film was prepared using a PMMA solution (3 wt \%, MW $950 \mathrm{k}$ ) in methoxybenzene (anisole). A relatively thick film was obtained by performing three spin-coating steps in sequence. In the first two steps, the solution was spin-coated at $500 \mathrm{rpm}$ onto the silicon wafer and then annealed for $2 \mathrm{~min}$ at $150{ }^{\circ} \mathrm{C}$. After a third spin-coating step at $500 \mathrm{rpm}$, the specimen was annealed for $10 \mathrm{~min}$ at $180{ }^{\circ} \mathrm{C}$.

The reference (calibration) sample was a plate of polystyrene (PS) approximately $1.2 \mathrm{~mm}$ thick (Goodfellow Corporation, Oakdale, PA). ${ }^{11}$ Values for the reduced storage modulus $E^{\prime *}$ and reduced loss modulus $E^{\prime \prime *}$ of the PS sample were measured with a Hysitron Bio Ubi VII nanoindenter (Hysitron Inc., Minneapolis, MN). In the measurements, a frequency sweep from 10 to $250 \mathrm{~Hz}$ was performed with a quasistatic load of $1000 \mu \mathrm{N}$ and a dynamic load of $20 \mu \mathrm{N}$. The experimental parameters used resulted in a contact depth greater than $400 \mathrm{~nm}$. The values of the reduced storage and loss moduli were determined to be $E^{\prime *}=(5.1 \pm 0.1) \mathrm{GPa}$ and 

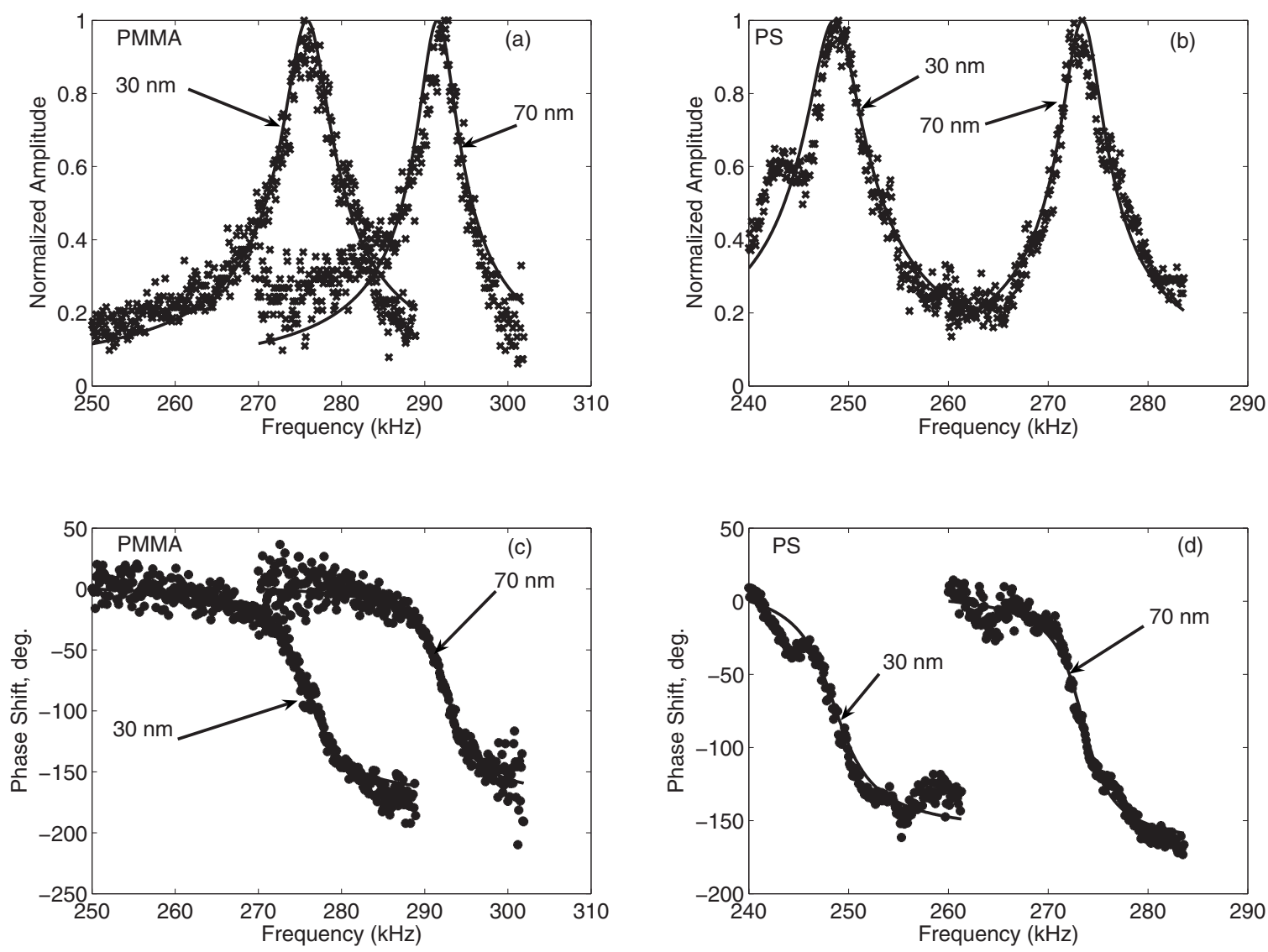

FIG. 4. Example of second mode response of the cantilever for the tip in contact with the PMMA film and the reference sample (PS). (a) and (b) shows the amplitude spectra for PMMA and PS, respectively. Respective phase spectra are shown in (c) and (d). The solid line shows the fit to the model given by Eqs. (11) and (12)

$E^{\prime \prime *}=(117 \pm 35) \mathrm{MPa}$, respectively. These values are consistent with those reported for PS in the literature. ${ }^{17,25,26}$ It is important to note that the accuracy of our approach relies on accurate knowledge of the properties of the reference sample. For a diamond indenter tip, $E=1140 \mathrm{GPa}$ and $\nu$ $=0.07$ resulting in $M_{t}=1145.6 \mathrm{GPa}$. The reduced modulus of the reference PS sample assuming contact with a silicon AFM tip is calculated to be $4.97 \mathrm{GPa}$ (assuming $M_{t}$ $=165 \mathrm{GPa})^{27}$

\section{RESULTS AND DISCUSSION}

Contact-resonance spectra were obtained for both the PMMA and PS samples at values of the cantilever deflection $d=30,50$, and $70 \mathrm{~nm}$. Assuming the nominal cantilever stiffness $k_{c}=1 \mathrm{~N} / \mathrm{m}$, this corresponds to applied static forces of $F_{N}=30,50$, and $70 \mathrm{nN}$, respectively. Spectra were acquired first on the PMMA film, then on the PS sample, and then once more on the PMMA film. Because the samples were homogeneous, significant changes in property with contact position were not expected. Figure 3 contains the response of the cantilever for the tip in contact with the PMMA film and the reference sample (PS). The figure shows the amplitude and phase spectra for the first flexural resonant mode of the cantilever. Similar plots for the second flexural mode are shown in Fig. 4. It can be seen that as the static deflection or applied load increases, the contact-resonance frequency increases. As described in the previous section, a nonlinear fit

TABLE I. Values for the parameters $a, b, \alpha$, and $\beta$ obtained from nonlinear fits of Eqs. (11) and (12) to the experimental contact-resonance amplitude data.

\begin{tabular}{|c|c|c|c|c|c|c|c|c|c|c|}
\hline \multirow[b]{2}{*}{ Sample } & \multirow[b]{2}{*}{ Trial number } & \multirow[b]{2}{*}{$d(\mathrm{~nm})$} & \multicolumn{3}{|c|}{ Mode 1} & \multicolumn{5}{|c|}{ Mode 2} \\
\hline & & & $a$ & $b\left(\times 10^{-3}\right)$ & $\alpha$ & $\beta$ & $a$ & $b\left(\times 10^{-3}\right)$ & $\alpha$ & $\beta$ \\
\hline & & 30 & 4.03 & 7.46 & 63.0 & 0.176 & 6.48 & 31.6 & 61.9 & 0.0598 \\
\hline \multirow[t]{3}{*}{ PMMA } & 1 & 50 & 4.07 & 5.07 & 79.8 & 0.188 & 6.67 & 34.1 & 78.9 & 0.0832 \\
\hline & & 70 & 4.07 & 5.00 & 79.2 & 0.183 & 6.74 & 28.2 & 87.0 & 0.0774 \\
\hline & & 30 & 4.00 & 8.53 & 53.1 & 0.147 & 6.18 & 34.7 & 42.4 & 0.0492 \\
\hline \multirow[t]{3}{*}{ PS } & 1 & 50 & 4.02 & 6.63 & 59.0 & 0.139 & 6.43 & 28.5 & 57.8 & 0.0508 \\
\hline & & 70 & 4.03 & 5.92 & 62.7 & 0.139 & 6.49 & 25.0 & 62.1 & 0.0476 \\
\hline & & 30 & 4.05 & 6.73 & 70.4 & 0.196 & 6.51 & 32.8 & 64.5 & 0.0646 \\
\hline \multirow[t]{2}{*}{ PMMA } & 2 & 50 & 4.07 & 5.05 & 78.5 & 0.181 & 6.66 & 30.8 & 78.4 & 0.0744 \\
\hline & & 70 & 4.07 & 4.98 & 78.5 & 0.179 & 6.69 & 26.9 & 81.4 & 0.0680 \\
\hline
\end{tabular}


TABLE II. Calculated values of the indentation storage modulus $M^{\prime}$ and loss modulus $M^{\prime \prime}$ assuming a flat tip $(m=1)$ or a hemispherical tip $(m=3 / 2)$.

\begin{tabular}{|c|c|c|c|c|c|c|c|c|c|}
\hline \multirow{3}{*}{ Data pair } & \multirow[b]{3}{*}{$d(\mathrm{~nm})$} & \multicolumn{3}{|c|}{ Mode 1} & \multicolumn{5}{|c|}{ Mode 2} \\
\hline & & & $M^{\prime}(\mathrm{GPa})$ & & $M^{\prime \prime}(\mathrm{MPa})$ & & $M^{\prime}(\mathrm{GPa})$ & & $M^{\prime \prime}(\mathrm{MPa})$ \\
\hline & & $m=1$ & $m=3 / 2$ & $m=1$ & $m=3 / 2$ & $m=1$ & $m=3 / 2$ & $m=1$ & $m=3 / 2$ \\
\hline \multirow{3}{*}{ PMMA1:PS1 } & 30 & 6.20 & 6.78 & 140 & 153 & 7.66 & 9.31 & 143 & 158 \\
\hline & 50 & 7.05 & 8.25 & 158 & 184 & 7.16 & 8.43 & 192 & 246 \\
\hline & 70 & 6.57 & 7.45 & 153 & 176 & 7.32 & 8.76 & 190 & 246 \\
\hline \multirow{3}{*}{ PMMA2:PS1 } & 30 & 6.94 & 7.38 & 157 & 181 & 7.98 & 9.93 & 153 & 176 \\
\hline & 50 & 6.94 & 7.38 & 153 & 176 & 7.16 & 8.43 & 171 & 206 \\
\hline & 70 & 6.53 & 7.32 & 151 & 172 & 6.83 & 7.87 & 167 & 200 \\
\hline
\end{tabular}

to Eq. (11) was performed on the amplitude curves in Figs. 3(a), 3(b), 4(a), and 4(b) to obtain values for the quantities $a$ and $b$. The fit is indicated in the figures by the solid lines. Fits to Eq. (12) were also used to obtain the solid lines in the phase curves of Figs. 3(c), 3(d), 4(c), and 4(d). It can be seen that the predicted response matches the experimental results very well.

Table I shows the values of $a, b, \alpha$, and $\beta$ determined for the PMMA film and PS sample. The calculated values of the indentation storage modulus $M^{\prime}$ and loss modulus $M^{\prime \prime}$ for the PMMA film are given in Table II. Values are shown for flatpunch contact $(m=1)$ and Hertzian contact $(m=3 / 2)$. Ideally, the values of $M^{\prime}$ and $M^{\prime \prime}$ calculated from the contact resonant frequency at a given cantilever deflection (static load) should be the same for both modes. The values that we report show slight variations in $M^{\prime}$ and $M^{\prime \prime}$ values for the two modes for all cantilever deflections. The difference in the values could be due to the fact that the cantilever beam is not perfectly rectangular as the model assumes. The assumption of perfectly clamped end may not have been the case for the particular cantilever used in these experiments. Also, the effect of the conical tip mass was neglected and the tip position was assumed to be at the far end of the cantilever. In the analysis, $L^{\prime}$ is taken to be zero after the realization that the adjustable tip position cannot be used as a fit parameter to obtain consistent values of $\alpha$ and $\beta$ for the two modes at all cantilever deflections (as done by others considering only the elastic case). When all of the results are averaged, for the PMMA film we obtain $M^{\prime}=(7.0 \pm 0.5) \mathrm{GPa}$ and $M^{\prime \prime}$ $=(160 \pm 16) \mathrm{MPa}$ using $m=1$, and $M^{\prime}=(8.1 \pm 0.9) \mathrm{GPa}$ and $M^{\prime \prime}=(190 \pm 30) \mathrm{MPa}$ for $m=3 / 2$. These values are roughly consistent with those obtained in previous studies of PMMA films with various contact methods. ${ }^{17,25,28}$ Several possible effects could explain any discrepancies between these and previously measured values. For instance, most of the previous work utilized tips with significantly larger radii and /or higher applied forces. The small size of the AFM tip could induce a contact strain sufficiently large to produce a nonlinear elastic response. ${ }^{29}$ In addition, the increase in the glass transition temperature $T_{g}$ as a function of tip load could be different for the two materials. This effect could make the small-contact-depth stiffness different, even though the bulk (large contact depth) stiffnesses are similar.

\section{SUMMARY}

In summary, this study demonstrates the feasibility of a contact-resonance force microscopy method (CRAVE) to de- termine viscoelastic material properties. The method is based on the AFAM approach originally developed for elastic materials. The forces between the AFM cantilever tip and the sample are modeled with a linear spring and dashpot. The complex wavenumbers of the problem are obtained by fitting a model curve to the experimental data. The wavenumbers are then related to the mechanical properties of the sample through the characteristic equation of the problem. To illustrate the technique, properties of a thin PMMA film were obtained using PS with known properties as the reference sample. The values of both the storage and loss modulus obtained were in agreement with those found using other techniques. Because this method can employ low forces and small contact depths, its benefit to the research and development of small-scale compliant materials is expected to be substantial.

\section{ACKNOWLEDGMENTS}

This work was supported by the National Science Foundation. The authors thank Ben Polly for valuable discussions.

${ }^{1}$ G. Binnig, C. F. Quate, and C. Gerber, Phys. Rev. Lett. 56, 930 (1986). ${ }^{2}$ U. Rabe, S. Amelio, M. K.-M. S. Hirsekorn, M. Kempf, M. Göken, and W. Arnold, Surf. Interface Anal. 33, 65 (2002).

${ }^{3}$ P. Maivald, H. J. Butt, S. A. C. Gould, C. B. Prater, B. Drake, J. A. Guriey, V. B. Eiings, and P. K. Hansma, Nanotechnology 2, 103 (1991).

${ }^{4}$ D. Liu, G. Benstetter, and E. Lodermeier, Thin Solid Films 436, 244 (2003).

${ }^{5}$ H. J. Butt, B. Cappella, and M. Kappl, Surf. Sci. Rep. 59, 1 (2005).

${ }^{6}$ M. Muraoka and W. Arnold, JSME Int. J., Ser. A 44, 396 (2001).

${ }^{7}$ M. R. Vanlandingham, S. H. McKnight, G. R. Palmese, R. F. Eduljee, J. Gillespie, and J. R. L. McCulough, J. Mater. Sci. Lett. 16, 117 (1997).

${ }^{8}$ A. Rosa-Zeiser, E. Weilandt, S. Hild, and O. Marti, Meas. Sci. Technol. 8, 1333 (1997)

${ }^{9}$ N. A. Burnham, A. J. Kulik, G. Gremaud, P. Gallo, and F. Oulevey, J. Vac. Sci. Technol. B 14, 794 (1996).

${ }^{10}$ P. Attard, J. Phys.: Condens. Matter 19, 473201 (2007)

${ }^{11}$ Commercial equipment and materials are identified only in order to adequately specify certain procedures. In no case does such identification imply recommendation or endorsement by the National Institute of Standards and Technology, nor does it imply that the materials or equipment identified are necessarily the best for the purpose.

${ }^{12}$ D. C. Hurley, M. Kopycinska-Müller, and A. B. Kos, JOM 59, 23 (2007).

${ }^{13}$ U. Rabe, S. Amelio, E. Kester, V. Scherer, S. Hirsekorn, and W. Arnold, Ultrasonics 38, 430 (2000).

${ }^{14}$ D. C. Hurley and J. A. Turner, J. Appl. Phys. 95, 2403 (2004).

${ }^{15}$ D. C. Hurley, M. Kopycinska-Müller, D. Julthongpiput, and M. J. Fasolka, Appl. Surf. Sci. 253, 1274 (2006).

${ }^{16}$ S. A. Syed Asif, K. J. Wahl, R. J. Colton, and O. L. Warren, J. Appl. Phys. 90, 1192 (2001).

${ }^{17}$ C. A. Tweedie, G. Constantinides, K. E. Lehman, D. J. Brill, G. S. Blackman, and K. J. Van Vliet, Adv. Mater. (Weinheim, Ger.) 19, 2540 (2007).

${ }^{18}$ U. Rabe, J. Turner, and W. Arnold, Appl. Phys. A: Mater. Sci. Process. 66, 
S277 (1998).

${ }^{19}$ J. A. Turner, S. Hirsekorn, U. Rabe, and W. Arnold, J. Appl. Phys. 82, 966 (1997).

${ }^{20}$ L. Meirovitch, Principles and Techniques of Vibrations (Prentice-Hall, Saddle River, NJ, 1997), p. 07458.

${ }^{21}$ L. Meirovitch, Fundamentals of Vibrations (Thomas Casson, Springfield, IL, 2001).

${ }^{22}$ U. Rabe, K. Janser, and W. Arnold, Rev. Sci. Instrum. 67, 3281 (1996).

${ }^{23}$ K. L. Johnson, Contact Mechanics (Cambridge University Press, Cambridge, 1985).
${ }^{24}$ R. M. Christensen, Theory of Viscoelasticity, 2nd ed. (Academic, New York, 1982).

${ }^{25}$ B. J. Briscoe, L. Fiori, and E. Pelillo, J. Phys. D 31, 2395 (1998).

${ }^{26}$ K. Miyake, N. Satomi, and S. Sasaki, Appl. Phys. Lett. 89, 031925 (2006).

${ }^{27}$ D. C. Hurley, M. Kopycinska-Müller, A. B. Kos, and R. H. Geiss, Adv. Eng. Mater. 7, 713 (2005).

${ }^{28}$ J. Zhou and K. Komvopoulos, Appl. Phys. Lett. 90, 021910 (2007).

${ }^{29}$ E. K. Dimitriadis, F. Horkay, J. Maresca, B. Kachar, and R. S. Chadwick, Biophys. J. 82, 2798 (2002). 